\title{
EVENTI DI DIPARTIMENTO 2019
}

18 febbraio. Ricordando Ettina Confalonieri. Tra impegno scientifico e passione civile. Sul tema: "Teoria politica, integrazione europea e studi di genere" intervengono Francesco Battegazzorre, Maurizio Ferrera, Marina Piazza. Sul tema "Studenti europei, cittadini del mondo" intervengono Gianni Vaggi, Ilaria Poggiolini, Ernesto Bettinelli.

5 marzo. Public Lecture di Gunter Gaublomme sul tema "Economic Diplomacy".

13 marzo. Presentazione del programma World in Progress e delle Borse Manera. Introduzione di Roberta Rabellotti. Presentazione di Stefania Paradisi.

12 marzo, 2 aprile, 11 aprile. Ciclo di conferenze: Pavia Silicon Valley 2019.

14 marzo. Seminario tenuto da Anna Martinelli sul tema: "Brevetti ed internazionalizzazione dell'attività innovativa nei paesi emergenti".

19 marzo. Conferenza tenuta da Marco Modenesi sul tema: "Écritures et réécritures de l'Amérique: Volkswagen Blues de Jacques Poulin".

26 marzo. Seminario tenuto da Andrea Morrison sul tema: "Migration and invention in the Us during the age of mass migration".

4 aprile. Cerimonia di consegna delle borse di studio Giovanni Manera. Francesco Montessoro, Gianluca Ferrittu e Gabrile Proietto hanno discusso sul tema "Il Myanmar alla ricerca di un modello di sviluppo sostenibile". Ha coordinato Roberta Rabellotti. Conclusioni di Enrica Chiappero.

12 aprile. Convegno sul tema: "L'anti-corruzione. Prospettive etiche, istituzionali e problemi di misurazione". Interventi di Michele Bocchiola, Nicoletta Parisi, Maria Pia Redaelli, Chiara Bardelli. Moderano Emanuela Ceva e Silvia Figini. Conclusioni di Alessandro Venturi. 
3 maggio. Presentazione del volume: "Lives in Transit. An Ethnographic Study of Refugees' Subjectivity across European Borders". Interviene l'autrice Elena Fontanari.

6 maggio. Seminario tenuto da Ferdi De Ville sul tema: "Naive no longer? The evolution of the EU's trade policy response to China's rise".

9 maggio. Seminario tenuto da Maria Silvia Vaccarezza e Federico Zuolo sul tema: "Eccessi di morale. Forme del moralismo e virtù correttive".

14 maggio. Seminario tenuto da Juan Carlos Castillo Sanchez sul tema: "Innovation Systems and Global Value Chains in Latin America".

21 maggio. Annual lecture 2019 on Human Rights and International Justice. Fatsah Ouguergouz interviene sul tema: "La protection juridique des personnes vulnérables: l'Afrique à l'avant-garde/The legal protection of vulnerable people: Africa at the forefront" e Fausto Pocar sul tema: "The case-law of international criminal Courts and Tribunals on the protection of vulnerable people".

23 maggio. Lezione di Gérard Chouin sul tema: "Can Africa's history be 'medieval'? Some thoughts on connectedness and deep memory in the Gulf of Guinea".

27 maggio. Ciclo di lezioni "Storie in corso". Lezione di Federica Ferrero sul tema: "Notabili, intermediari e nazionalisti: l'élite di Tetuán tra continuità e trasformazione (1945-1960)". Lezione di Carlotta Marchi sul tema: "La Sanusiyya e la nascita della Libia indipendente. Il ruolo della Confraternita islamica nel processo di costruzione dello Stato tra gli anni Quaranta e Cinquanta".

29 maggio. In occasione delle Elezioni Europee del 26 maggio Francesco Battegazzorre e Silvia Illari organizzano l'incontro sul tema: "Dopo le elezioni europee". Ne discute Paola Bilancia.

3-4 giugno. Seminario di ricerca sul tema: "Mobilità e contenimento dei migranti tra Africa e Mediterraneo: le politiche europee e le risposte africane". Interventi di Corrado Tornimbeni, Eliana Augusti, Arrigo Pallotti, Antonio M. Morone, Luca Ciabarri.

27 giugno. Presentazione del libro "La Lega di Salvini. Estrema destra al governo". Andrea Zatti e Guido Legnante ne discutono con l'autore Gianluca Passarelli.

17- 26 settembre. Summer School di Studi dell'Africa e dell'Asia 2019. Seminari di cultura, storia e politica dell'Africa e dell'Asia.

26 settembre. Seminario tenuto da Fabrizio Rossi sul tema: "Il go- 
verno instabile. Per una periodizzazione politico-istituzionale dell' Italia liberale (1861-1922)".

30 settembre. Inaugural Public Lecture 2019/2020: World Politics and International Relations. David Baldwin ha tenuto una lezione sul tema: "Thinking about Power in International Relations".

7 ottobre. Seminario di Changwei Chen sul tema: "China and the World Order. Status quo or Chinese Style?". Introduce Enrica Chiappero. Intervengono Matteo Dian, Simone Dossi, Roberta Rabellotti. Discute Axel Berkofsky. Modera Marco Clementi.

8 ottobre. Presentazione del volume "Persone e mondi. Azioni individuali e ordine internazionale". Dino Cofrancesco, Lorenzo Ornaghi, Enrica Chiappero, Francesco Battegazzorre ne discutono con l'autore Angelo Panebianco.

8 ottobre. Presentazione del volume "Periferie dell'Occidente. Italia e Sudafrica nell' ordine liberale 1795-1996". Stefano Allovio, Arianna Arisi Rota, Pierluigi Valsecchi ne discutono con l'autore Rocco W. Ronza.

10 ottobre. Lectio magistralis tenuta da Staffan de Mistura sul tema: "Complexity in International Conflicts: Lessons from the Past and for the Future".

15 ottobre. Presentazione del volume "Terrore e terrorismo. Saggio storico sulla violenza politica". Arianna Arisi Rota, Francesco Marone, Fabio Rugge, Pierluigi Valsecchi ne discutono con l'autore Francesco Benigno.

16 ottobre. Incontro con Benedetta Tobagi e Giorgio Boatti su: "Piazza Fontana prima e dopo".

22 ottobre. Seminario di Meriem Dhouib sul tema: "Cohésion et éclatement dans la littérature maghrébine d'expression française".

12 novembre. Presentazione del libro "Risorgimento. Un viaggio politico e sentimentale". Marina Tesoro, Paolo Colombo, Mino Milani dialogano con l'autrice Arianna Arisi Rota.

12 novembre. Seminario sul tema: "Economia dello sviluppo e riduzione della povertà. Riflessioni sul premio Nobel 2019”. Partecipano Giovanna Prennushi e Marinella Leone. Introduce Enrica Chiappero.

13 novembre. Seminario di approfondimento sul tema della violenza contro le donne. Lezioni di Silvia Illari (La normativa a protezione delle donne vittime di violenza), Anna Rita Calabrò (I numeri della violenza sulle donne), Lea Melandri (Il sogno d'amore e la violenza invisibile). 
14 novembre. Lezione di Sara Franzone sul tema: "Commercio internazionale e decarbonizzazione. Le sfide dell'industria europea dell'acciaio". Introduce Jacques Ziller.

14 novembre. Seminario di Piero Maffettone sul tema: "A Theory of International Toleration". Intervengono Enrico Biale, Giulia Bistagnino, Federica Liveriero, Federico Zuolo.

14 novembre. Lectio magistralis di Germano Maifreda sul tema: "Storia: di chi? Passato europeo, passati subalterni".

20 novembre. Tavola rotonda sul volume: "La passione per la Repubblica. Studi dedicati a Marina Tesoro" a cura di Arianna Arisi Rota e Bruno Ziglioli. Partecipano Silvio Beretta, Barbara Bracco, Salvatore Lupo.

21 novembre. Seminario di Andrés Lazzarini sul tema: "Growth, Policies, Inequality and Crisis: the political-economic pendulum in Argentina and Brazil during 2003-2019 (with some reflection on Latin America)". Discussant: Marco Missaglia.

4 dicembre. Lezione di Elias Zigah sul tema: "Renewable Energy a rationale for African Integration".

5 dicembre. Lezione di Elias Zigah sul tema: "Regional Integration and Power Nexus in Africa, a case study of ECOWAS and the West Africa Power Tool".

5-6 dicembre. Convegno sul tema: "Paris 1919 and the Global South" in collaborazione con il Dipartimento di Studi Storici dell' Università degli Studi di Milano.

10 dicembre. Lezione di Benedetta Tobagi sul tema: "I terrorismi politici in Italia. Origini, caratteristiche e sviluppi”. 